\title{
Statistical significance of rank regression
}

\begin{abstract}
Rank regression, which is quite simple to use some form of monotonic relationship between $\mathrm{X}$ and $\mathrm{Y}$. Since the rank regression is a nonparametric approach so there are essentially no confidence interval, hypothesis tests, prediction intervals, and interpretation of regression coefficients. In this article, we proposed a bootstrap statistical significance measure of the rank regression by formulating a bootstrap interval for the rank regression parameters. If the rank regression parameters from the original data are not within the bootstrap interval, the rank regression parameters are considered significance. Numerical examples show that the merit of using this proposed bootstrap interval.
\end{abstract}

Keyword: Bootstrap; Rank regression; Statistical significance. 